\title{
Museum factors affecting the ageing process of organic materials: review on experimental designs and the INVENVORG project as a pilot study
}

\author{
Petros Dellaportas ${ }^{1 \dagger}$, Effie Papageorgiou $^{2 \dagger}$ and Georgios Panagiaris ${ }^{3 *}$
}

\begin{abstract}
The most common way followed for the determination of degradation mechanisms of organic materials is the application of physiochemical methods after accelerated aging. In recent years, there has been a remarkable research activity towards the development of alternative decay determination methods in organic materials based on statistics. We first present a survey of European research projects in this area together with summaries of their results. Second, we describe the ongoing project entitled 'Investigation of the environmental factor effects on organic materials constituting the natural and cultural heritage' in which statistics plays a crucial role. In particular, we present a statistical methodology that is based on a designed experimental and inferential protocol which will guide the research throughout the project. By providing basic statistical and design concepts, we present guidelines to design statistical protocols that are necessary for scientifically sound quantitative experiments.
\end{abstract}

Keywords: Preventive conservation; Museum environment; Organic materials; Experimental design; Chemometric studies

\section{Introduction}

A primary concern of a conservator is the safeguarding of the aesthetic, historical, structural and material integrity of the objects he/she conserves (E.C.C.O. 2002 [1]). This is achieved by diagnosing the state of preservation and by planning and applying the methods of preventive and interventive conservation. The assessment of the preservation state of objects of cultural interest has an important benefit to conservation and delineates the adequate preventive measures that would lead to better controlled storage and exhibition conditions; this would also secure future possible studies of the objects. The overall condition assessment of such objects is highly desirable; however, it is also extremely difficult, if not impossible, to achieve. Investigation techniques targeting to marginal states (i.e. state of least degradation, or "initial" state, and

\footnotetext{
*Correspondence: gpanag@teiath.gr

${ }^{\dagger}$ Equal contributors

3 Department of Conservation of Antiquities \& Works of Art, Technological

Educational Institute of Athens, GR-12210 Aegaleo, Greece

Full list of author information is available at the end of the article
}

state of the higher expected degradation) could offer a remedy to the problem. However, this approach actually offers only a small part of the needed information due to the dynamic and sometimes contradicting nature of the degradation process. Moreover, the degradation extent may not be uniform across the macroscopic, microscopic and the molecular level. Few reports offer a hint to such inter-relation and it it clear that more work is needed in this direction. The COST CMST Action D42 Chemical Interactions between Cultural Artefacts and Indoor Environment (EnviArt) has brought together researchers and experts from various European countries and disciplines working on the above mentioned topic. According to the most recent outcomes of this Action (October 2010) [2]:

"The description of the state-of-art showed that research on the chemical interactions between cultural artifacts and the indoor environment and standardization is performed already at the international level, but still needs serious efforts for coordination. Only a close multidisciplinary 
cooperation will ensure that our current collection of cultural artifacts will be accessible for future generations".

Remarkable research results have been provided on the chemical effects of the most common types of fine particulate matter in typical indoor materials in museums and libraries $[3,4]$. The investigation of the degradation mechanisms of the materials constituting the cultural and natural heritage, despite the progress made, has a long way still to go. The most common way followed for the determination of these mechanisms is the application of physicochemical methods after accelerated ageing. The disadvantage of this practice is the inability to control the effect of the combination of degradation factors in real time and in real conditions as it happens in burial environment, in museum environment or in open-air conditions. This problem is getting even worse in the case of organic materials that are characterized by a variety in structure and composition, and that are extensively sensitive in many environmental factors, abiotic and - mainly- biotic. The need for understanding the decay process -especially of these organic materials with industrial use (i.e. paper, wood) - resulted to the standardization of diagnostic techniques and of the accelerated ageing protocols (ASTM 1993 [5]; ASTM 1995 [6]; ASTM 1996 [7]; ASTM 2002a [8]; ASTM 2002b [9]). Although this standardization, it is no coincidence that in recent years it has been noticed a remarkable research activity towards the development of alternative decay determination methods in organic materials based on statistical elaboration of analytical data obtained experimentally.

The application of statistics in the cultural heritage area is very limited. To demonstrate that their adoption is important, we review in section "Review" the most important international research projects that investigate the effects of the influence of the indoor environmental factors on organic materials constituting cultural heritage. We present research projects focusing on the collection and processing of chemical analysis. It is stated that the role of statistic is not only the processing of these chemical measurements (chemometrics), but also the overall survey design including its initial experimental design. In this framework, a bibliographic reference to important research results have made use of statistical methods and techniques in the field of conservation studies, is presented in section "Review". In section "INVENVORG Project: concept and objectives" we describe the ongoing project INVENVORG (Investigation of the environmental factor effects on organic materials constituting the natural and cultural heritage) which may be viewed as a pilot study for the research area of conservation sciences. In particular we emphasize the need to adopt experimental designs and inferential protocols which guide the research throughout projects. Such statistical protocols are necessary for scientifically sound quantitative experiments.

\section{Review}

\section{Chemometrics}

In recent years, the application of statistical techniques and methods has been expanded in heritage studies area due the development of chemometric methods. According to the generic definition, accepted by the International Chemometrics Society (ICS), chemometrics is the science of correlating, by statistics-mathematic techniques, of measurements conducted on a chemical system or process, its state being in given conditions or at a given time. Although the term of chemometrics was introduced in 1972 by the Swede Svante World and the American Bruce R. Kowalski, the operative definition of chemometrics, stated by practitioners, was established in 1986 and presented in the editorial of the first issue of Chemometrics and Intelligent Laboratory Systems journal, in the following sentence:

"Chemometrics is that discipline in the field of chemistry which uses methods of mathematics and statistics to formulate or select experimental plans and procedures of physical-chemical analysis, in view of extracting maximum information by algorithmic processing of data obtained as a result of studies conducted at laboratory or industrial level" (Deselnicu 2010 [10]).

A better, concise definition exists on the home page of the chemometrics and intelligent laboratory systems:

"Chemometrics is the chemical discipline that uses mathematical and statistical methods to design or select optimal procedures and experiments, and to provide maximum chemical information by analysing chemical data" [11].

This method is based on statistical elaboration of analytical data from groups of existent objects and aims at the correlation of the factors that -potentially- are responsible for their decay and at the determination of the critical factor that can be considered as catalyst for their preservation (Malea, 1999 [12]; Larsen et al., 2002 [13]; Bechmann and Larsen, 2007 [14]; Zervos, 2007 [15]; Deselnicu, 2010 [10]; Malea et al., 2010 [16]; Malea et al., 2011 [17]).

The pragmatic purpose of chemometrics is establishing quantitative relationships between related or logically derived forms of one and the same product or of a series of products in different states, as well as numeric descriptions of those states connected in physicalchemical processes clearly defined in time, resorting to chemical or instrumental analytical determinations. In order to achieve this goal, the data obtained experimentally are processed through advanced techniques of 
mathematical statistics, numeric analysis and information theory, employing highly optimized algorithms and software applications.

Usually, chemometrics is resorted to in situations where theoretical knowledge of physical-chemical features of processes and states is not advanced enough to completely describe or correctly solve problems arisen in the study of physical-chemical systems. From this point of view, chemometric techniques can be considered to be semi-empirical, because they offer nondeterministic and sometimes stochastic models, or non-parametric mathematical relations to describe physical-chemical systems. The objective of chemometric techniques is that of highlighting complex and non-obvious relationships (hidden) between a large number of variables associated with studied systems, resorting to statistic-mathematic models capable of describing states of physical-chemical systems, at a given time or in their dynamic.

Another important issue is the evaluation of materials and methods. Statistical science can offer new approaches to infer from data in different disciplines, taking into account the uncertainty present in such data. Such an approach is not error free. Typically there is missing information, since data are rarely complete. Moreover some of the data may contain errors due to systematic or other reasons.

Statistical application in conservation of cultural heritage is not often found in literature. In 1998, the Getty Institute created an elementary guide for statistical application for workers in the field. Statistics as a basic tool in that field is developed later by including the contributions of members of the Research Team of this project. Mitchell et al. [18], supported previous publication results indicating that attenuated total reflectance-Fourier transform infrared (ATR-FTIR) is a useful tool for the examination of objects containing polymers. In [18], it was shown that polymers could be characterised in object fragments that were not specially prepared and without the use of a spectral library. Principal component analysis (PCA) was shown to be a useful tool for the unambiguous identification of cellulose acetate, cellulose nitrate, polycarbonate or polyurethane polymers in historical plastics with different additives, plasticisers or age. More excitingly, even though spectral features were similar for new and aged samples of polyurethane, PCA was able to discriminate between samples of foam that had been treated by heat $\left(50^{\circ} \mathrm{C}\right.$ for $24 \mathrm{~h}$ or $144 \mathrm{~h}$ ) or by exposure to light, although more results for other polymeric materials are required to support this proof of concept study.

Statistical methods have been only occasionally used in studies of paper aging and the effect of the environment in its properties. They have been primarily focused in the estimation of the effective time of the paper and the application of the Arrhenius equation. The study of multiple samples with statistical methods has led to different ideas about the paper maintenance and identified interesting problems related to the design of experiments, highlighted by the importance of storage conditions. Kinetic models have identified correlations between different characteristics leading to interesting insights on the mechanisms underlying the phenomenon $[19,20]$. The advantage of applying statistical methods has also made a huge impact on the study of the mechanism of attrition for other materials like leather and parchment. Finally, statistical methods have been used to examine the effect of the museum environment on cultural heritage objects [15].

\section{Experimental design - real case studies}

Parchment manuscripts are priceless records of the beliefs, ways of living and governing that form the roots of the European civilization. It is known that parchment is a collagen-based biomaterial endowed with great stability, strength, resilience and exceptional longevity. However, it is sensitive to environmental damaging factors and especially to humidity associated with temperature variations Larsen et al. 2005 [21], Bowden et al. 2002 [22] and Hansen et al. 1991 [23].

The preservation and the methods of conservationrestoration of parchment and leather are a significant concern among conservators. There is a wide variety of objects made of leather and parchment in the world collections and many of them surprise by their beauty or unusual technique. The significant sensitivity of these materials to physical, chemical and mechanical factors motivate conservators to elaborate new and safe methods for their conservation, and to ensure stable storage conditions.

The role of experimental design in most of real case studies is becoming increasingly important. For example, recently Elena Badea et al. 2010 [24], applied in new parchments accelerated ageing protocols, established within the compass of the EU project IDAP (Improved Damage Assessment of Parchment) [25], at the Centre de Recherches sur la Conservation des Documents Graphiques, Paris, using a humidity and temperature test chamber) and a dry oven. The accelerated ageing procedures consisted in consecutive steps of the following treatments:

1. Exposure to gaseous solutions of chemical pollutants (50 ppm of $\mathrm{NO}_{2}, 50 \mathrm{ppm}$ of $\mathrm{SO}_{2}, 25 \mathrm{ppm} \mathrm{SO}+$

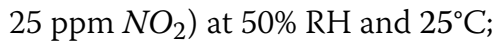

2. Exposure to chemical pollutants, in the same condition as in (1), after a pre-treatment by irradiation with visible light $(1.7 \times 105 \mathrm{~lx})$;

3. Exposure to chemical pollutants, in the same condition as in (1), after a pre-treatment by heating at $100^{\circ} \mathrm{C}$ in dry oven; 
4. Exposure to chemical pollutants, in the same condition as in (1), after a pre-treatment by both irradiation with visible light $(1.7 \times 105 \mathrm{~lx})$ and dry heating $\left(100^{\circ} \mathrm{C}\right)$.

The duration of one ageing step was 2 weeks for pollutant exposure, 4 hours for visible light irradiation and 2 days for dry heating.

Other examples of accelerated ageing experiments as tools in the study of deterioration and deteriorative factors under this project, are given by Frederique Juchauld et al. [26], Marie Vest et al. [27] and Rene Larsen et al. [28].

As an organic material, papyrus is subject to chemical processes induced by acid, air, and water. Papyrus, unlike newsprint, lightens rather than darkens with light; in fact, sun-bleaching is one conservation treatment that has been applied to enhance legibility ( Leach and Tait 2000 [29]). However, photo-oxidation breaks down cellulose, and papyri exposed to continuous light may disintegrate completely. One accelerated aging experiment found light to be the single greatest threat to papyrus (Flieder et al. 2001 [30]).

Other organic materials such as wood, cellulosic fabrics, paper and the bone have been studied by investigators. Seli et al. [31] (1998) studied the kinetics of degradation of cellulosic fabrics (cotton, linen) by artificial aging at constant temperature $\left(123-192^{\circ} \mathrm{C}\right)$, as well as in combination to the effect of light $(280-380 \mathrm{~nm})$ in the temperature range of $50-85^{\circ} \mathrm{C}$ and $\mathrm{RH} 10 \%$ and $70 \%$. The natural aging of cellulosic materials (high purity paper, cotton, raw and bleached flax, viscose) was simulated by thermal oxidation in air at the constant temperature and the synergetic effect of light.

Wood as a natural organic material is directly affected by biotic and abiotic factors. The most extensively studied factors are the heat, the radiation and the relative humidity (Stamm et al. [32], 1960; Skaar, 1988 [33]; Green et al. 1999 [34]; Rivers and Umney, 2005 [35]; Yidiz et al. [36], 2006; Welzbacher \& Rapp, 2007 [37]; Yokoyama et al. [38], 2009.

Of all the materials stored in museums, archives and libraries, paper is the most extensively studied. As noted above, there exist numerous studies concerning the impact of the environment on the ageing of paper which have been critically reviewed in several publications (Feller et al. 1986 [39]; Fellers et al. 1989 [40]; Emsley \& Stevens 1994 [41]; Feller 1994 [42]; Shahani et al. 2001 [43]; Zervos 2010 [20]; Area \& Cheradame 2011 [44]). Most of these studies include the exposure of samples of various types of contemporary and historic paper to controlled atmospheres inside ageing chambers. These experiments have utilized the full range of all possible combinations of temperatures and relative humidities. The cycling of relative humidity and temperature has also been studied (Shahani et al. 1989 [45]).
There have been many studies conducted regarding the ageing and thermal effects on bone (Child, 1995 [46]; Grupe, 1995 [47]; Hedges and Millard, 1995 [48]; Klinken and Hedges, 1995 [49]; Evershed et al., 1995 [50]; Richards et al., 1995 [51] ; Panagiaris et al., 1996 [52]; Beisaw, 1998 [53]; Richter \& Kalsbeek, 2001 [54]; Kalsbeek \& Richter, 2006 [55]). The environmental parameters that have been mostly investigated in the above-mentioned studies that play a key role in bone degradation are temperature and humidity.

For all these organic materials, conducted research to identify the critical factors affecting their aging. This makes to the design of experiments a very important contribution in this research area.

\section{European research projects}

Within Europe, research on the effects of air pollutants on the degradation of organic materials constituting cultural heritage has been carried out for decades in specialized museums, universities, research institutes and by environmental agencies, which managed to establish several collaboration projects at the European level. Within the EU Third Framework Programme (FP3) (1990) two research projects initiated the field on interactions of air pollutants and organic cultural heritage; namely the EU STEP "Leather project" and the STEP "Paper project" [56]. In 1996, within FP4, the second EU project on leather deterioration was completed. Other EU projects relating to the subject "indoor air pollution monitoring and chemical interactions" are the following: IDAP, AER, IMPACT, LIDO, MIMIC, MASTER, MIP, ERA, SENSORGAN, PAPYLUM, SURVENIR, INKCOR, PAPERTREAT, PARELA, CARAMEL, MULTIENCODE, VIDRIO, MODHT, PROPAINT, and MEMORI [57]. As already mentioned, IDAP [25] has been dedicated to damage assessment of parchment (damage due to environment). The IDAP project, Improved Damage Assessment of Parchment 2002-2005, was funded by the EC under the fifth framework program: Energy, environment and sustainable development (EESD). AER, [58] has been concerned with the assessment of indoor air quality at museums in order to discover the impact of the building and artificial air-control on the quality of the collection. IMPACT, LIDO, MIMIC and MASTER developed forecasting models, advanced sensor technology and prototypes using for instance lasers to monitor the indoor impact on cultural objects of pollution, exposure to light or fluctuations in humidity and temperature caused by human presence in particular. The IMPACT-project, aimed to develop models of the behavior of pollution such as sulphur dioxide, nitrogen dioxide and ozone, [59] on museum and building materials. Both MIMIC and MASTER were related to dosimeters for the assessment of the museum environment. The LiDo-project, focused in the 
impact of light on artworks. Within the EU network MIP Working Group 4 is dedicated to preventive conservation. The problem to be solved by the network is to respond collectively to specific threats to the European paper-based cultural heritage. These risks include the deterioration, conservation and storage of paper and ligno-cellulosic objects that have been affected by metal-tannin corrosion throughout, many of which are stored in archives, libraries and museums. Furthermore, the ERA project (Environmental Research for Art Conservation) [60] has to be mentioned here. This project was studying the effect of environment (light, thermal and pollution) on the deterioration of physical and chemical conditions of paintings. Also within this project, spectroscopic techniques such as NIR and mass spectrometry were applied to understand the effects of the environment on the deterioration. Because of the extensive number of EU projects, collaborations on an individual basis also exist and networks such as the IAQ-network are operational. Participants in these projects took an active role during the preparation of the COST Action [2]. The CEN TC 346 (Standardization Conservation of Cultural Property) can be seen as the main existing network seeking collaboration with the COST Action. The CEN TC 346 Working Group 4 dealing with standardization of indoor environment is especially interested in gaining knowledge of the chemical interactions between cultural heritage objects and air pollutants. The International Council of Museums and the Committee for Conservation with their working groups on preventive conservation, chemical analyzes and scientific research on art objects would be interested in establishing a close cooperation with the COST Action.

SENSORGAN developed new sensors for monitoring and detecting harmful environments on pipes and wooden parts of organs. PAPYLUM and SURVENIR created non-invasive tools to analyse the state of paper documents, while INKCOR developed ways to neutral - ise the acidity of iron gall ink, used on paper for centuries in Europe and stored in countless but often damaged archives. The follow-on FP6 project, PAPERTREAT, is evaluating existing techniques in the con - text of commercialisation. The main objective of PARELA project is to establish a laser-cleaning system suitable for accurate and safe restoration of paper objects, with focus on the incorporation of a reliable control system.

It is known that changes are taking place in the composition of the atmosphere and in the resulting impact of multipollutants on the environment and land. Cultural heritage is particularly vulnerable to such changes. The results of the EC-financed CARAMEL project [61], wound up in 2003, showed that carbonaceous particulate has now become the main damage factor on the architectural and archaeological heritage. Measurements performed in a number of European cities (Milan, Rome,
Paris, London and Seville), both in the atmosphere and on monuments, have proved that the blackening of surfaces is increasing and is attributable to automobile traffic. The data were obtained thanks to an innovative methodology for the measurement of the carbon component in stone materials, monitoring campaigns in the proximity of monuments (such as Milan Cathedral and S. Maria del Fiore in Florence) and assessments of the visual perception of monument blackening by the public (tourists and inhabitants). It has also been demonstrated how the composition of the damage layers responsible for the blackening of the architectural heritage is changing, which imposes the need for suitable cleaning techniques. The results achieved underscore the urgency of adopting air quality thresholds that are compatible with the preservation of the cultural heritage, in order to avoid irreversible damage, escalating restoration costs, and the gradual disappearance of our monuments under a cloak of blackness.

Advanced laser measurement technique in one operational device for structural assessment of a wide range of issues like cracks, voids, detachments were investigated in the MULTIENCODE project for structural recognition purposes. In addition, within EC VIDRIO project [62] a study focused on the improvement of methods and technological systems in order to control and reduce damage of stained glass protected with glazing and not protected, exposed to climatic influences, air pollution and biodeterioration was developed. The environmental impact on the stained glass in target sites (i.e. Sainte Chapelle, Paris and Cathedral of Cologne) was particularly studied as a consequence of the daily and seasonal cycles of the climatic and other environmental variables (e.g. visitors, heating and air-conditioning systems, infiltration of polluted air from outside). In particular the local climate (air temperature, dew point, relative and specific humidity, ventilation, radiation) and the internal microclimate concerning the stained glass under study (glass temperature, spread above the dew point, condensation on the panel and time of wetness, ventilation) was monitored with automatic devices and special intensive field surveys. The final goal is to give a final judgment on the goodness of the double glazing as a solution for a better conservation of stained glass from all the point of view (physical chemical and biological), to provide a methodology for the best management of these buildings and identify the thresholds of danger to preserve stained glass.

The project (MODHT) has produced an objective testing scheme for damage assessment with which conservators will be able to assess and track the condition of the tapestries within their care. This is especially important because of the invisibility of fibre damage: changes at the molecular level have consequences for the physical strength of the tapestry. A new understanding of degradation has been obtained and changes in overall strength 
and colour can be described for the first time in chemical terms. The state of the fibre is of vital importance for the overall mechanical integrity of the tapestries and will determine how they will withstand being transferred to other locations for display. The proven correlations between amino acid analysis, molecular weight (silk) and tensile strength (among other markers) of silk and wool confirms the value of applying these analytical results to future conservation strategy. Useful correlations and inter-relationships have been established which will benefit conservators. For example, the analytical techniques could be used to prioritise tapestries for interventive conservation treatment, and also to justify the level of intervention or support required in order to stabilise and display a tapestry. - See more at the project's website [63].

Finally, the PROPAINT project, "Improved protection of paintings during exhibition, storage and transit", (2007 - 2010) is implemented within the EU 6th Framework Programme, Priority 8.1 "Policy oriented research" [64]. Measurements of air pollution and climate inside and just outside of microclimate frames with and without paintings in 12 museum sites and 1 frame making company in Europe have been performed. It was shown that levels of volatile organic acids should also be considered, and this is currently being followed up in the new MEMORI project. The MEMORI project, "Measurement, Effect Assessment and Mitigation of Pollutant Impact on Movable Cultural Assets. Innovative Research for Market Transfer", is implemented within the EU 7th Framework Programme. The project started on November 1, 2010 and has a duration of 36 month. The aim of the MEMORI project is to provide the conservation market with innovative, non destructive, early warning technology for easy assessment of environmental impact on indoor cultural heritage. In addition a preventive strategy to secure the conservation of movable cultural assets in protective enclosures has been developed. Also, the current pollution situation and management of perhaps materials emitting unsuitable or corrosive gases were explored. In addition, correlations between the expenses for collection care (preventive conservation; monitoring of the environmental conditions $(\mathrm{T} / \mathrm{RH} / \mathrm{HV})$; pollutant measurement (airborne pollutants and dust/particles/soiling) and the possible influencing factors were analyzed. For this propose univariate and bivariate frequency distributions were used. Its study design is based on the interviews were organized by an online questionnaire and an automatically readable pdf-questionnaire [65].

\section{INVENVORG Project: concept and objectives}

We provide in this section a summary description of the ongoing project INVENVORG. In contrast to the inorganic materials of cultural heritage, organic materials have been subjected to less systematic research. For this reason, in the framework of the research project INVENVORG, it has been planned to carry out the artificial aging of non treated organic materials (parchment, woolen textile, bone, wood and paper) under individual environmental factors such as temperature $(\mathrm{T})$, relative humidity $(\mathrm{RH})$, ultraviolet (UV) and visible radiation, sulfur dioxide (SO2) and nitrogen oxides (NOx). These factors were judged to be the most important. At the same time it is planned to attempt a behavior study of these materials in real museum environments in which the air gases, the climatic factors, the air particles and the bioaerosol will be measured. The plan is to achieve (i) Modeling the non-treated organic materials' decay mechanisms; (ii) Correlating the ageing of non-treated organic materials with real data; (iii) Evaluation of the analysis and diagnosis methods (i.e. assessment of the preservation state); and (iv) Development of good practice artificial ageing that can be used for testing and control. The quantitative and qualitative condition assessment of aged and non-aged samples, as this is determined through the study of the structure and of the chemical composition of organic materials, will be achieved by modern diagnostic techniques (macroscopic, microscopic, nanoscopic, chemical analytical and mechanical), aiming at recording qualitative and quantitative changes in physical, chemical or even intangible features. It is therefore considered necessary to include in this study, representative samples from various organic materials instead of an in depth investigation of only one kind of material. The obtained data will be gathered in a comprehensive, searchable database, which structures complex analysis results in a simple, easily accessible and intelligible manner in view of applying statistics techniques. The outcomes of the project would be utilized for the standardization of the appropriate conditions for the preventive conservation of organic materials in museums and libraries.

The project started in March 2012 and it consists of 7 Work Packages, scheduled to be completed in 48 months. The research groups participating in this project are Technological Educational Institute of Athens (coordinator), National Technical University of Athens, University of Patras, Foundation of Research and Technology of Patras, University of Ioannina, University of Crete, Technological Educational Institute of Crete, Technological Educational Institute of Piraeus, Athens University of Economics and Business, Danish Royal Academy of Fine Arts. The project is operating under the action 'Thalis' co-funded by the Greek Ministry of Education and the European Union (National Strategic Reference Framework -NSRF).

The first results of the project relating to one of the five test materials, namely paper, have been presented at the international conference "3rd International Conference on Integrated Information held in Prague, Czech Republic, September 5-9, 2013". The presentation entitled: 
"Experimental design for the investigation of the environmental factors effects on organic materials (Project INVENVORG). The case of paper", by S. Zervos, K. Choulis and G. Panagiaris which was part of Workshop Session entitled: "Conservation And Preservation Issues In Libraries And Archives", has been published in the open access scientific journal "Procedia - Social and Behavioral Sciences" [66].

An innovative part of this project is the use of statistical design of experiments. We believe that this is a crucial part of the scientific study so we provide, in the next subsections, some basic definitions and guidelines of statistical design of experiments. We first provide in section "Statistical methods for the analysis of data" some introductory statistical concepts and we outline in section "Design of experiments" the basic ingredients of the design of experiments together with the guidelines given to all participants of project INVENVORG.

\section{Statistical methods for the analysis of data}

A statistical experiment is characterized by the treatments and experimental units, the way treatments are assigned to units, and the responses that are measured. The response or dependent variable is the variable of interest to be measured in the experiment. Factors are those variables whose effect on the response is of interest to the experimenter. Factor levels are the values of the factor utilized in the experiment. The treatments of an experiment are the factor - level combinations utilized. An experimental unit is the object on which the response and factors are observed or measured. A designed experiment is an experiment in which the analyst controls the specification of the treatments and the method of assigning the experimental units to each treatment. An observational experiment is an experiment in which the analyst simply observes the treatments and the response on a sample of experimental units. The completely randomized design is a design in which treatments are randomly assigned to the experimental units or in which independent random samples of experimental units are selected for each treatment.

In most cases, the results of an experiment are analyzed with the use of analysis of variance (ANOVA). The effects of a single factor are termed main effects. An oneway (one factor) ANOVA therefore has only a single main effect. In a design with more than one factor, such as a two-way design with two factors, we can also explore interaction effects. An interaction between two factors (a two-way interaction) is present if the effects of the factors are not independent. In general researchers will ignore a non-significant two-way interaction and interpret the two main effects which are considered to be independent of each other. If a two-way interaction is significant it may not make sense to interpret the main effects on their own because they represent 'average' effects of a factor which are known to vary between levels of the other factor. A main effect is easy to interpret when there are no significant interactions involving it. In the presence of a significant higher-order interaction, the lower-order interactions and corresponding main effects are hard to interpret by themselves. The size of the effects must be detected and the noise of the process (how much variation it has) will dictate how much replication is needed. Confounding occurs when the experimental controls do not allow the experimenter to reasonably eliminate plausible alternative explanations for an observed relationship between independent and dependent variables.

Given $k$ factors with 2 levels each there are $2^{k}$ possible combinations. Designs including all levels are called $2^{k}$ factorial designs. To estimate all of them with replications, becomes prohibitively expensive. A $2^{k-f}$ design is a design that drops some combinations in the interest of cost, but introduces some confounding to the model. For instance if $f=1$ then the experimenter only uses half of the combinations. This means that it may be impossible to "separate" some low order interactions effects with higher order interactions (i.e. they will be confounded). Which effects are confounded depends on the defining relation of the fractional study. Good defining relations leave as many low order interactions (including main effects) estimable as possible. Objectives of Fractional Factorials are (i) to reduce the number of required runs and (ii) to screen out insignificant factors in the initial stages of experimentation. This can be done without substantial loss in information if higher-order interactions can be assumed to be negligible.

Planning an experiment properly is very important in order to ensure that the right type of data and a sufficient sample size and power are available to answer the research questions of interest as clearly and efficiently as possible. A clear definition of the details of the experiment makes the desired statistical analysis possible, and almost always improves the usefulness of the results. The overall data collection and analysis plan considers how the experimental factors, both controlled and uncontrolled, fit together into a model that will meet the specific objectives of the experiment and satisfy the practical constraints of time and money. The data collection and analysis plan provides the maximum amount of information that is relevant to a problem by using the available resources most efficiently. Understanding how the relevant variables fit into the design structure indicates whether the appropriate data will be collected in a way that permits an objective analysis that leads to valid inferences with respect to the stated problem. The desired result is to produce a layout of the design along with an explanation of its structure and the necessary statistical analysis. 
There are at least two problems that may occur in a study: missing values and small data sample. We should link the cases of missing values met in Conservation of Cultural Properties with the missing data mechanisms namely: a) Missing Completely at Random (MCAR) b) Missing at Random (MAR) and c) Non-Ignorable (NI) or Missing Not at Random (MNAR). For example, missing data of archaeological organic materials may be due to loss of material, to insufficient or inadequate of diagnostic methods, or/and to incomplete documentation (loss of information) during the steps that take place between the excavation and the transportation to the Laboratory. We have to determine which of the above mentioned cases can be considered as MACR, MAR or MNAR (Malea et al., 2011 [17]).

Regarding the handling of the small data, we could borrow some ideas from the biostatistics literature. The European Commission (Health and Consumer Protection Directorate-General) considers that a disease is rare if its prevalence is lower than 50 cases per 100,000 habitants. Hence, according to this definition, rare diseases will have very low observed cases in low populated areas. This definition could also be applied in archaeological organic materials that can be only rarely found due to their vulnerability in degradation factors. Wakefield et al. [67] describe some methods for the detection of disease clusters and show some examples of their use. Besag and Newell [68] divide the types of methods for the detection of disease clusters in two groups: General methods and Focused methods. Another type of methods is that of Scan methods. Equally, relevant methods should be developed for the statistical elaboration of the data derived from organic materials. These data could be linked with "charts" of burial or museum environments. Additionally, we can say that sample size is specified by pre-defining the standard deviation of the material, the cost and available budget and the materials availability.

\section{Design of experiments}

Since the early work of Ronald Fisher, (Box, 1980 [69]), the concept of scientific design of experiments has become a necessary ingredient in efficient scientific experiments. In this respect, all large scientific bodies throughout the world have implemented precise structures for the types of experiments they perform. In particular, medical communities that first introduced experimental designs through clinical trials, have reached a point in which no top-level publication is achieved without a previously publicized experimental protocol. Since credibility of the experimental outcome is of primary interest in all serious and legitimate studies, such self-control of the scientific community is absolutely necessary. We outline below the guiding principles followed by all participants of INVENVORG for the construction of experimental protocols before they start their experiments. In the near future it will be reported the experimental protocol constructed by using these guidelines.

Background research and personal experience of the researcher should lead to clear statement of the reasons for conducting the experiment. There must be some description of all relevant studies published on the subject. Ideally, although usually hard to achieve, a researcher should exploit past studies but avoid biases caused by the results obtained by them. A researcher should have a clear view of the type of information that will be collected and its value in the relevant scientific community.

Specific objectives and statement of research questions and hypotheses should be clearly, and as simply as possible, stated in advance of the experiment. The hypotheses must be clearly testable, and, ideally, clearly disproved. Statistical science teaches us that evidence can only be used to state that a hypothesis is not true (with some probability of error) but we can never have enough evidence to state that a hypothesis is true under all possible conditions. It is noted here that a hypothesis is not required for an experiment to be well designed since an experiment might be taken place just to investigate how a response reacts under various experimental conditions. In this process of the design, there must be some sense of all the factors and variables that will be involved in the experiment.

The design of the study should first describe the type of the study. A study may be observational, interventional, descriptive, analytic, cross-sectional, longitudinal, controlled, non-controlled, etc. The design should include the sampling scheme, the research population and the duration, the sample size, the factors and their levels to be used, the statistical design related to research limitations such as the variability of each response measurement and the cost estimation.

There must be detailed information of the methodology that will be used in the experiment. Interventions such as description of material/methods or training of researchers or information to laboratory technicians should be planned in advance. Procedures that describe the data collection process are essential for the reliable statistical analysis, so they must be described in advance of the study. Moreover, there is some need to plan ahead the way and the time measurements will be taken or observations will be made. The randomization process which is essential for the credibility of the statistical analysis must be described and care should be taken so that biases do not occur. Finally, timings of assessment and group meetings are essential. A flow diagram is usually provided with all the above issues stated across time of the project.

Data handling, coding and storing should be part of the experimental design. The sample sizes and the power of the study including the reasoning for their choice, the level 
of significance, procedures to deal with missing data or errors in measurement should be clearly outlined. There must be clear statement of how the data will be analyzed. Although many statistical tests may take place at the end of the study, these will be only complimentary. The main statistical tests that answer core questions of the study should be certainly chosen and stated in the experimental design protocol.

There must be some indication of how the study advances current knowledge on the issues under question, what its impact may be and how the results will be utilized. Each member of the team should have clear and well defined responsibilities and deliverables.

In all experimental designs many things may go wrong. There might be controls that have not been samples, so the resulting data is meaningless since there is nothing to be compared against. The sample size may be relatively small to be reliable so when cost of increasing the sample size is not an issue, the greater the sample size the better.

Bad randomization is also crucial because adds biases in the results that can not be easily handled retrospectively (although Statisticians have tools to reduce the problem so finally the efficiency of the sample is decreased but the data may not be useless). Researcher bias should be identified and diminished but it usually occurs. Cause and effect issues are delicate and usually misinterpreted. Correlations do not imply causes and hidden factors (confound effects) may have been ignored in the experiment.

Finally, it is emphasized the one and only absolute ingredient that makes an experiment scientific: The experiment process should be repeatable. Any conclusions are totally useless if the experimental conditions cannot be constructed again. This, clearly, distinguishes science from pseudo-science.

\section{Conclusions}

We have provided a review of an area of particular interest in conservation studies, namely the determination of museum factors affecting the aging process of organic materials. Our review has been based on the following pillars.

We first reviewed a series of recent European projects and pinpointed their results. Then, we focused in the important issue of the use of statistical methodology adopted in this area, and claimed that more widespread use of statistical techniques is needed. Finally, by focusing in the ongoing project INVENVORG we described how a statistical protocol based on both inferential procedures and design of experiments has been adopted and will be used throughout the project duration.

We strongly believe that the field of conservation studies needs to adopt a scientific approach in designing the related experiments and we do hope that our article will be of valuable help to all researchers in the area.

\section{Competing interests}

The authors declare that they have no competing interest.

\section{Authors' contributions}

PD and GP designed research. PD and EP contributed to the role of statistics and design of experiments whereas GP contributed to review of conservation studies, chemometrics, and the project INVENVORG. All authors read and approved the final manuscript.

\section{Acknowledgements}

This research has been co-financed by the European Union (European Social Fund - ESF) and Greek national funds through the Operational Program "Education and Lifelong Learning" of the National Strategic Reference Framework (NSRF) - Research Funding Program: THALES. Reinforcement of the interdisciplinary and/or inter-institutional research and innovation with the possibility of attracting high standard researchers from abroad through the implementation of basic and applied excellence research.

\section{Author details}

${ }^{1}$ Department of Statistics, Athens University of Economics and Business, 76 Patission Str., GR-10434 Athens, Greece. ${ }^{2}$ Department of Medical Laboratories, Technological Educational Institute of Athens, GR-12210 Aegaleo, Greece. ${ }^{3}$ Department of Conservation of Antiquities \& Works of Art, Technological Educational Institute of Athens, GR-12210 Aegaleo, Greece.

Received: 30 May 2013 Accepted: 12 December 2013

Published: 16 January 2014

\section{References}

1. E.C.C.O. Professional Guidelines 1. 2002. [http://www.ecco-eu.org/ about-e.c.c.o./professional-guidelines.html] (Accessed 15/8/2011).

2. Chemical Interactions between Cultural Artefacts and Indoor Environment (EnviArt). [http://www.cost.eu/domains_actions/cmst/ Actions/Chemical_Interactions]

3. Grau-Bove J, Strlic M: Fine particulate matter in indoor cultural heritage: a literature review. Heritage Sci 2013, 1:8.

4. Smolik J, Maskova L, Zikova N, Ondrackova L, Ondracek J: Deposition of suspended fine particulate matter in a library. Heritage Sci 2013, 1:7.

5. ASTM D2244 - 11: Standard practice for calculation of color tolerances and color differences from instrumentally measured color coordinates. ASTM, Book Standards 2011, 6:12. doi:10.1520/D2244-11.

6. ASTM D198 - 13: Standard test methods of static tests of lumber in structural sizes. ASTM, Book Standards 2013, 4:27. doi:10.1520/D0198.

7. ASTM E313 - 10: Standard practice for calculating yellowness and whiteness indices from instrumentally measured color coordinates. ASTM, Book Standards 2010, 6:6. doi:10.1520/E0313-10.

8. ASTM D6819-02: Standard test method for accelerated temperature aging of printing and writing paper by dry oven exposure apparatus (Withdrawn 2011). ASTM 2007. 4 doi:10.1520/D6819-02R07.

9. ASTM D6833-02: Standard test method for accelerated pollutant aging of printing and writing paper by pollution chamber exposure apparatus (Withdrawn 2011). ASTM 2007. 4. doi:10.1520/D6833-02R07.

10. Deselnicu DC: Applications of statistical methods in investigating heritage leather items. Revista Leather and Footwear J, eds. INCDTPDivision: Leather and Footwear Research Institute March 2010, 10(1):13-30.

11. Chemometrics and Intelligent Laboratory Systems. [http://www. journals.elsevier.com/chemometrics-and-intelligent-laboratory-systems]

12. Malea $E$, Watkinson, DE: Contribution of analytical methods in the estimation of the condition of waterlogged archaeological leather. In Advanced Study Course 1999, 6-10 July 1999, School of Conservation, Royal Academy of Fine Arts, Copenhagen, Denmark, European Commission Directorate-General XII, Environment and Climate Programme, Preprints; 1999:249-259.

13. Larsen R, Poulsen DV, Odlyha M, Kurt N, Wouters J, Puchinger L, Brimblecombe $P$, Bowden D: The use of complementary and comparative analysis in damage assessment of parchments. In Microanalysis of Parchment. Edited by Larsen R. London: Archetype; 2002:165-179.

14. Bechmann DJ, Larsen R: The IDAP website - Data collection and sharing of knowledge. In School of Conservation, The Royal Academy of 
Fine Art, Improved damage assessment of parchment IDAP, Assessment, data collection and sharing of knowledge, Research Report no 18, Copenhagen, European Commission-Directorate General for Research I-Environment. Edited by Larsen R. Luxembourg: Office for Official Publications of the European Communities; 2007:13-16

15. Zervos $S$ : Evaluating treatments of paper using statistically valid test methods. Part II: Experimental setup and protocol. Restaurator 2007, 28(4):256-288.

16. Malea E, Vogiatzi T, Watkinson DE: Assessing the physical condition of waterlogged archaeological leather. In 11th ICOM-CC WOAM WG Conference in Greenville. North Carolina USA; May 24-28, peer review paper; 2010:571-592.

17. Malea $E$, Papageorgiou E, Panagiaris $G$ : Investigation of the degradation mechanisms of organic materials: from accelerated ageing to chemometric studies. In Proceedings of the International Conference on Integrated Information (IC-ININFO 2011). Edited by Georgios AG, Damianos PS. Kos Island Greece; 29th October - 3rd November; 2011:280-284. [http://history.icininfo.net/2011/FileStore/procs_INFO_2011.pdf]

18. Mitchell G, France F, Nordon A, Tang PL, Gibson LT: Assessment of historical polymers using attenuated total reflectance-Fourier transform infra-red spectroscopy with principal component analysis. Heritage Science 2013, 1:28.

19. Zou X, Uesaka T, Gurnagul N: Prediction of paper permanence by accelerated aging I. Kinetic analysis of the aging process. Cellulose 1996, 3:243-267.

20. Zervos S: Natural and accelerated ageing of cellulose and paper: $A$ literature review. In Cellulose: Structure and Properties, Derivatives and Industrial Uses. Edited by Lejeune A, Deprez T. New York: Nova Publishing; 2010:155-203

21. Larsen R, Poulsen DV, Juchauld F, Jerosch H, Odlyha M, de Groot J, Wess TJ, Hiller J, Della Gatta G, Badea E, Masic A, Boghosian S, Fessas D: Damage assessment of parchment: complexity and relations at different structural levels. In Proceedings 14th Triennal Meeting ICOM-CC vol. I. London: The Hague James\&James; 2005:199-208.

22. Bowden D, Brimblecombe P: The thermal response of parchment and leather to relative humidity changes. In Microanalysis of Parchment. Edited by Larsen R. London: Archetype Publications Ltd.; 2002:63-72.

23. Hansen EF, Lee $S N$, Sobel $H$ : The effects of relative humidity on some physical properties of modern vellum: implications for the optimum relative humidity for the display and storage of parchment. In AIC-The Book and Paper Group Annual 10. Washington: The American Institute for Conservation; 1991. [http://cool.conservation-us.org/coolaic/ sg/bpg/annual/v10/]

24. Badea E, Budrugeaca P, Della Gattab G, Miuc L, Comanescua A: A DSC study of deterioration caused by environmental chemical pollutants to parchment, a collagen-based material. Thermochimica Acta 2010, 500:51-62.

25. Improved Damage Assessment of Parchment 2002-2005. [http://www.re-ad.dk/kaks/en/publications/the-idap-website]

26. Juchauld F, Jerosch H, Dif K, Ceccarelli R, Thao S: Effects of two pollutants ( $\mathrm{SO} 2$ and NO2) on parchment by analysis at the molecular level using mass spectrometry and other techniques. Correlation with differential scanning calorimetry and visual assessment results. Research Report No 2007, 18:59-66.

27. Vest M, Jacobsen J, Larsen R: Accelerated ageing effect of heat and relative humidity. Research Report No 2007, 18:67-68.

28. Larsen R, Vestergaard Poulsen D, Minddal K, Dahlstrom N, Fazlic N: Damage of parchment fibres on the microscopic level detected by the micro hot table (MHT) method. Research Report No 2007, 18:69-72.

29. Leach B, Tait J: Papyrus. In Ancient Egyptian Materials and Technology. Edited by Paul TN, lan S. New York: Cambridge University Press; 2000:227-253

30. Flieder F, Delange E, Alain Duval A, Leroy M: Papyrus: the need for analysis. Restaurator 2001, 22(2):84-106.

31. Selli E, Beltrame PL, Testa G, Bonfatti AM, Rossi E, Seves A: Kinetic studies on the accelerated aging of cellulosic materials. Die Angewandte makromolekulare Chemie 1998, 257:63-69.

32. Stamm AJ, Baechler RH: Decay resistance and dimensional stability of five modified woods. Forest Products $J 1960,10: 22-26$

33. Skaar C: Wood-water relations. In Springer Series In Wood Sciences. Berlin: Springer-Verlag; 1988:283.
34. Green DW, Winandy JE, Kretschmann DE: Mechanical properties of wood. In Wood Handbook: Wood as an Engineering Material. Madison, WI USDA Forest Service, Forest Products Laboratory; 1999:463. Gen. Tech. Rep. FPL.-GTR-113.

35. Rivers S, Umney N: Conservation of Furniture. Oxford: Elsevier, Butterworth-Heinemann; 2005

36. Yidiz S, Gezer ED, Yildiz UC: Mechanical and chemical behaviour of spruce wood modified by heat. Build Environ 2006, 41:1762-1766.

37. Welzbacher CR, Rapp A: Durability of thermally modified timber from industrial-scale processes in different use classes: Results from laboratory and field tests. Wood Mater Sci Eng 2007, 2:4-14.

38. Yokoyama M, Gril J, Matsuo M, Yano H, Sugiyama J, Clair B, Kubodera S, Mistutani T, Sakamoto M, Ozaki H, Imamura M, Kawai S: Mechanical characteristics of aged Hinoki wood from Japanese historical buildings. CR Physique 2009, 10:601-611.

39. Feller RL, Lee SB, Bogaard J: The kinetics of cellulose deterioration. In Historic Textile and Paper Materials. Conservation and Characterization. Advances in Chemistry Series 212. Edited by Needles HL, Zeronian SH. Washington: American Chemical Society; 1986:329-347.

40. Fellers C, Iversen T, Lindstrom T, Nilsson T, Rigdahl M: Ageing/degradation of paper, a literature survey. In FoU-projektet for papperskonserering. Stockholm: FoU-projektet for papperskonservering; 1989.

41. Emsley AM, Stevens GC: Kinetics and mechanisms of the low-temperature degradation of cellulose. Cellulose 1994, 1:26-56

42. Feller RL: Accelerated Aging: Photochemical and Thermal Aspects: Marina del Rey, The Getty Conservation Institute. Ann Arbor: Edwards Bros.; 1994.

43. Shahani C, Lee SB, Hengemihle FH, Harrison G, Song P, Sierra ML, Ryan CC, Weberg N: Accelerated aging of paper: I. Chemical analysis of degradation products. II. Application of Arrhenius relationship. III. Proposal for a new accelerated aging test: ASTM research program into the effect of aging on printing and writing papers. Washington, DC: Library of Congress; 2001.

44. Area MC, Cheradame H: Paper aging and degradation: recent findings and research methods. BioResources 2011, 6(4):5307-5337.

45. Shahani $\mathrm{CJ}$, Hengemihle $\mathrm{FH}$, Weberg $\mathrm{N}$ : The effect of variations in relative humidity on the accelerated aging of paper. In Historic textile and paper materials II. Conservation and characterization. ACS Symposium Series 410. Edited by Zeronian SH, Needles HL. Washington: American Chemical Society; 1989:63-80.

46. Child AM: Microbial Taphonomy of archaeological bone. Stud Conserv 1995, 40:19-30

47. Grupe G: Preservation of collagen in bone from dry, sandy soil. J Archaeological Sci 1995, 22(N.2):193-200.

48. Hedges REM, Millard AR: Bones and groundwater: towards the modeling of diagenetic processes. J Archaeological Sci 1995, 22(N.2):155-164.

49. Klinken GJ, Hedges REM: Experiments on collagen-humic interactions: speed of humic uptake, and effects of diverse shemical treatments. J Archaeological Sci 1995, 22(N.2):263-270.

50. Evershed RP, Turner-Walker G, Hedges REM, Tuross N, Leyden A: Preliminary results for the analysis of lipids in ancient bone. J Archaeological Sci 1995, 22(N.2):277-290.

51. Richards MB, Sykes BC, Hedges REM: Authenticating DNA extracted from ancient skeletal remains. J Archaeological Sci 1995, 22(N.2):291-300

52. Panagiaris $\mathrm{G}$, Gioni $\mathrm{P}$, Malea $\mathrm{E}$, Papadimitriou $\mathrm{M}$, loakimoglou $\mathrm{H}$ : Estimation de l' etat de preservation d' ossements archeologiques en Grece, basee sur la presence des elements calcium et azote: Premiers resultats. In Third International Conference on Bone Diagenesis. Paris, France; 4-7 March 1996:54

53. Beisaw AM: Differential preservation and recovery: taphonomy Of bone preservation at the Thomas/Luckey site. Ashland. NY: State University of New York; 1998.

54. Richter J, Kalsbeek N: Chemical and physical changes observed in heated bones. In Accepted abstract for 4th Mummy Congress; September 4-10 Nuuk 2001

55. Kalsbeek N, Richter J: Preservation of burned bones: an investigation of the effect of temperature and pH on hardness. Stud Conserv 2006, 51(2):123-138. 
56. Larsen R: Summary discussion and conclusion. In STEP Leather Project. Edited by Larsen R. Denmark: Copenhagen, Royal Danish Academy of Fine Arts; 1994:165-180.

57. Preserving our heritage, Improving our environment, Volume II, Cultural heritage research: FP5, FP6 and related projects.

[http://ec.europa.eu/research/environment/pdf/ 20years_cultural_heritage_vol2_en.pdf]

58. Gysels K, Delalieux F, Deutsch F, Van Grieken R, Camuffo D, Bernardi A, Sturaro G, Busse HJ, Wieser M: Indoor environment and conservation in the Royal Museum of Fine Arts, Antwerp, Belgium. J Cultural Heritage 2004, 5(N.2):221-230.

59. Blades N, Kruppa D, Cassar M: Development of a web-based software tool for predicting the occurrence and effect of air pollutants inside museum buildings. In ICOM Committee for Conservation, 13th Triennial Meeting, Rio de Janeiro, 22-27 September. London: Maney Publishing; 2002:9-14.

60. Odlyha M, Boon JJ, van den Brink O, Bacci M: Environmental research for art conservation (ERA). J Thermal Anal 1997, 49(N.3):1571-1584.

61. CARAMEL book: Air pollution and cultural heritage. (invited ed.: $C$. Saiz-Jimenez), 15 papers from the CARAMEL Consortium, Balkema; 2004

62. Publications from Vidrio European Project. [http://www.isac.cnr.it/ vidrio/publications.htm]

63. Monitoring of damage to historic tapestries (The 'MODHT' project). [http://www.hrp.org.uk/aboutus/whatwedo/collectionscare/ monitoringthedamagetohistorictapestries\#sthash.aXtnwbW7.dpuf]

64. PROPAINT. [http://propaint.nilu.no/Results/tabid/596/language/en-US/ Default.aspx]

65. MEMORI. [http://www.memori-project.eu/memori.html]

66. Zervos S, Choulis K, Panagiaris G: Experimental design for the investigation of the environmental factors effects on organic materials (Project INVENVORG). The case of paper. 3rd International Conference on Integrated Information (IC-ININFO). Elsevier; 2013. [http://users.teiath.gr/szervos/_objects/docs/zervos+houlis+ panagiaris_experimental_design_author_ver.pdf]

67. Wakefield JC, Kelsall JE, Morris SE: Clustering, cluster detection and spatial variation in risk. In Spatial Epidemiology. Methods and applications, chapter 8. Edited by Elliot P, Wakefield JC, Best NG, Briggs DJ. Oxford: Oxford University Press; 2000:128-152.

68. Besag J, Newell J: The detection of clusters in rare diseases. J R Stat SoC SerA 1991, 154:143-155.

69. Box JF: R. A. Fisher and the design experiments 1922-1926. Am Stat 1980, 34(1):1-7.

\section{doi:10.1186/2050-7445-2-2}

Cite this article as: Dellaportas et al: Museum factors affecting the ageing process of organic materials: review on experimental designs and the INVENVORG project as a pilot study. Heritage Science 2014 2:2. 